\title{
Photo-electron Emission at Mercury-Dimethylsulfoxide Solution Interface and Following Electrochemical Process*
}

\author{
Kazuo YAMASHITA** and Hideo IMAI**
}

\begin{abstract}
The photo-electrochemical process of dimethylsulfoxide was studied by using a dropping mercury electrode irradiated by ultraviolet light. It was assumed that the photo-emitted electrons from the irradiated dropping mercury electrode were thermalized and solvated in solution. Dimethylsulfoxide was then reduced by the solvated electron to methylsulfinylcarbanion around the irradiated dropping mercury electrode. The quadratic dependence of the photocurrent on the concentration of dimethylsulfoxide was proveded experimentally in the non-aqueous medium. The law of five halves was found to hold in relation to the field effect on the photocurrent. The apparent threshold potential was evaluated to be $-0.66 \mathrm{~V}$ vs. NCE by applying the double layer correction. It was shown that the real free energy of the solvation of the electron, $U_{\mathrm{e}-\mathrm{s}}$, could be expressed in terms of the values of the threshold potential, the energy quantum of the incident light and the work function relevant to the transfer of the electron from the electrode mercury into solution, involving the work function of the electron from the metal into vacuo and the Volta potential difference at the metal-solution interface. The value of $\left\{U_{\mathbf{e}^{-\mathbf{s}}}-\left(V_{\mathrm{m}-\mathrm{s}}\right)_{\phi_{\mathrm{pzc}}}\right\}$, where $\left(V_{\mathrm{m}-\mathrm{s}}\right)_{\phi_{\mathrm{pzc}}}$ is the Volta $\mathrm{p}$. d. between the metal and solution at the potential of zero charge, in dimethylsulfoxide was estimated to be about a half of that in water. Finally, the discrepancy between the values of $U_{\mathrm{e}-\mathrm{s}}$ determined by means of the photoelectrochemical emission method and by the radiolysis study was discussed.
\end{abstract}

\section{Introduction}

Investigations on the physico-chemical processes at a micro-electrode irradiated by ultra violet light have been developed in this decade. The interest in this field lies in the fact that this photo-electrochemical technique is promising for the study of fast chemical reactions of $e_{\text {aq }}$ with scavengers.

On the occurrence of the photocurrent observed at the irradiated micro-electrode three hypotheses have been proposed hitherto. Hey. rovsky") has assumed that the reaction proceeds through the charge transfer layer composed of the irradiated electrode and the depolarizer. Berg $^{2)}$ has suggested the excitation of the ir-

* A part of this paper was presented at the U.S.Japan Co-operative Science Seminar held in March 1973 in Tokyo.

** Department of Environmental Sciences, Faculty of Integrated Arts and Sciences, Hiroshima University (Hiroshima 730) radiated electrode and coined the term of the "hot electrode". On the contrary, Barker et $a{ }^{3} .^{3}$ have assumed that the photo-emitted electrons from the irradiated micro-electrode are partly thermalized and deposited as the solvated electrons in solution around a certain distance from the electrode surface, and then the solvated electrons are either captured by the scavenger ions or molecules, or they go into the selfdecay*1 except the electrons returning to the electrode. Supposing that the photocurrent flows according to the progress of the scavenging reaction, they have also showed that the photocurrent should be linearly proportional to the square root of the scavenger concentration. A

*1 The self-decay of the hydrated electrons has been studied in the radiation chemistry ${ }^{4,5}$ ) and it proceeds as follows; $e^{-}{ }_{\text {aq }}+\mathrm{H}_{2} \mathrm{O} \rightarrow \mathrm{H}+\mathrm{OH}^{-}$aq or $2 e^{-}{ }_{\text {aq }} \rightarrow$ $\mathrm{H}_{2}+2 \mathrm{OH}^{-}\left(k=1.1 \cdot 10^{10} M^{-1} \mathrm{sec}^{-1}\right)$. The decay to proceed either by the pseudo-first order reaction or by the bimolecular decay depends upon the concentration of the hydrated electrons. 
similar and more general theory on the photocurrent has been developed by the present authors ${ }^{6)}$ by treating the semi-infinite diffusion problem coupled with the scavenging reaction. The field effect on the photo-electron emission in solution was first treated by Delahay and Srinivasan $^{7)}$ on the basis of Fowler's theory of the photo-electron emission in vacuo. Delahay et $a l{ }^{8)}$ have also discussed the potential barrier at the electrode-solution interface for the photoelectron emission. Later, Brodsky, Gurevich and Sheberstov ${ }^{9}$ have treated the problem on the basis of the quantum mechanics and derived the law of five halves, that is, the proportionality of the photocurrent to the field strength to the power of five halves. Further, Brodsky et al. ${ }^{9}$, Pleskov and Rotenberg ${ }^{10)}$ and Rotenberg et al. ${ }^{11)}$ have pointed out the effect of the increasing potential difference in the diffuse double layer in a more diluted electrolyte solution, and Bomchil et al. ${ }^{12}$ ) have extensively studied the double layer effect.

In this paper the quadratic dependence of the photocurrent on the dimethylsulfoxide (DMSO) concentration and the law of five halves on the field effect are discussed from the experimental results obtained in DMSO or in the propylene carbonate (PC) solution containing DMSO, using the dropping mercury electrode (DME) irradiated by the ultraviolet light.

\section{Experimental}

The measurement of the photocurrent was carried out in a pyrex glass cell with the DME. A saturated or normal calomel electrode (SCE or NCE) provided with a salt bridge of the $\mathrm{PC}$ or DMSO solution was used as the reference electrode.

The current flowing through the cell at a given electrode potential, which was controlled by a Shimadzu Model PS-2 potentiostat, was recorded by a San-ei Galvanograph Recorder Model HR-101.

A 500 watts xenon lamp, Ushio Type UXL $500 \mathrm{D}$, was used for the irradiation through a Toshiba UV-D 25 filter. The wavelength of the incident light was $\sim 350 \mathrm{~nm}$, and the measurement was carried out by irradiating the one side of the DME without altering at all the optical alignment.

The differential capacity of the DME-solution interface was measured by an impedance bridge. The assembly was composed of two standard resistors of $150 \Omega$, a series circuit of a decade capacitor $(1.1 \mu F$ in 5 stages $)$ and a decade resistor ( $1.1 \mathrm{k} \Omega$ in 4 stages), and the cell circuit. The cell was of a three-electrode system, and the electrode potential was controlled by a potentiometer. An inductor of $50 \mathrm{H}$ was used to apply the $d$. $c$. potential from the potentiometer circuit to the DME. The sinusoidal wave $(1 \mathrm{kHz}, 50 \mathrm{mV})$ from a Yokogawa Hewlett-Packard Model 3300 A function generator was used for the impedance bridge, and the output of the bridge was observed by an oscilloscope, Iwasaki Model SS-5302 with a plug-in unit, Model SP-01H-A.

DMSO was distilled twice under a reduced pressure, and the middle fraction of $n_{\mathrm{D}}^{20}=1.475$, was collected and stored in an atmosphere of pure dried nitrogen. It was redistilled just before the preparation of the test solution. Propylene carbonate used as the solvent was purified by distilling twice under a reduced pressure in the presence of $p$-toluensulfonic acid, and the fraction of $n_{\mathrm{D}}^{20}=1.412$, was collected and stored in an atmosphere of pure nitrogen. Sodium perchlorate used as the supporting electrolyte was of $c$. $p$. reagent grade of Kanto Chemicals Co., and was dried at $110^{\circ} \mathrm{C}$ for a day and stored in a desiccator using $\mathrm{P}_{2} \mathrm{O}_{5}$ at least for one week. The concentration of the supporting electrolyte in the prepared solution was $0.1 M$ unless otherwise noted.

A running water jacket was used to control the cell temperature within the change of $\pm 0.15^{\circ} \mathrm{C}$. All experiments were carried out at $25^{\circ} \mathrm{C}$.

\section{Results and Discussion}

\subsection{Photocurrent}

In accordance with Barker's reaction model ${ }^{3>}$ we assumed that the electrons emitted into the solution from the irradiated electrode are thermalized and solvated at a certain distance in solution apart from the electrode surface, partly returning to the electrode or going into the self-decay. The solvated electron thus 
formed reacts with a scavenger in solution and the photocurrent flows according to the progress of the scavenging reaction.

A feature characterizing the photocurrent from the usual electrolytic current can primarily be attributed to the difference of the sites of the charge transfer (reducing process), that is, in the case of the photo-electrochemical process the charge transfers in a homogeneous zone in solution, while it transfers at a heterogeneous electrode-solution interface in the case of the usual electrode process. Accordingly, in the photo-electrochemical process the electrode potential is no longer significant as the electrolytic reduction potential ${ }^{* 2}$, while it affects the photocurrent in relation to the field effect on the photo-electron emission.

The photo-electrochemical processes are schematically shown as follows;

$$
\begin{aligned}
& \text { metal (electrode) }+h \nu \longrightarrow(\text { metal) })^{+}+e^{-} \\
& e^{-}+\text {solvent } \longrightarrow e^{-} \text {solv } \\
& e^{-} \text {solv }+S \text { (scavenger) } \longrightarrow S^{-}
\end{aligned}
$$

Reaction (1) denotes the photo-electron emission, Reaction (2) is the deposition of the solvated electrons in solution, and Reaction (3) the scavenging reaction.

The behavior of the solvated electrons corresponding to the above mentioned reaction model can be expressed by the semi-infinite diffusion equation coupled with the scavenging chemical reaction "), namely;

$$
\frac{\partial C_{\mathrm{e}}}{\partial t}=D_{\mathrm{e}} \frac{\partial^{2} C_{\mathrm{e}}}{\partial x^{2}}-k_{\mathrm{s}} C_{\mathrm{s}} C_{\mathrm{e}}
$$

where $C^{\prime} \mathrm{s}$ are the concentration of the respective species, $D_{\mathrm{e}}$ the diffusion coefficient of the solvated electron, and $k_{\mathrm{s}}$ the rate constant of Reaction (3). It is noteworthy that in Eq. (4) the origin of the $x$ axis is defined at the plane ( $\delta$ ) of the deposition of $e^{--}$solv.

The initial and boundary conditions for $\mathrm{Eq}$. (4) are as follows;

$$
\begin{array}{ll}
C_{\mathrm{e}}=0 & \text { at } x=0, t=0 \\
C_{\mathrm{e}}=C_{\mathrm{e}}{ }^{\delta} & \text { at } x=0, t=t \\
\frac{d C_{\mathrm{e}}}{d x}=0 & \text { at } x=r, t=t
\end{array}
$$

where $C_{\mathrm{e}}{ }^{\delta}$ denotes the concentration of $e^{-}{ }_{\text {solv }}$ at

*2 The standard oxidation potential of $e^{-}$aq on the hydrogen scale was determined as great as $2.7 \mathrm{~V}$ from the radiation chemistry studies ${ }^{18}$. the deposition plane, $r$ the distance where $e^{-}$solv is depleted by the scavenging reaction.

The approximate solution of Eq. (4) for the steady state, $\partial C_{\mathrm{e}} / \partial t=0$, gives the photocurrent, $i_{\mathrm{p}}$, as follows;

$$
\begin{aligned}
i_{\mathrm{p}}= & -F D_{\mathrm{e}}\left(\frac{d C_{\mathrm{e}}}{d x}\right)_{x=0} \\
& =F D_{\mathrm{e}} C_{\mathrm{e}}^{\delta} \sqrt{\frac{k^{\prime}}{D_{\mathrm{e}}}} \tanh \sqrt{\frac{k^{\prime}}{D_{\mathrm{e}}}} \cdot r
\end{aligned}
$$

where it is assumed that $C_{\mathrm{e}} \ll C_{\mathrm{s}}$ and $k^{\prime}=k_{\mathrm{s}} C_{\mathrm{s}} \fallingdotseq$ constant.

When the scavenging reaction rate is sufficiently high, $\left(k^{\prime} / D_{\mathrm{e}}\right)^{1 / 2} r \longrightarrow \infty$, and $\tanh \left(k^{\prime} / D_{\mathrm{e}}\right)^{1 / 2} \cdot r$ can be approximated as unity. Then, one obtains the following expression from Eq. (6);

$$
i_{\mathrm{p}}=F D_{\mathrm{e}} C_{\mathrm{e}}^{\delta} \sqrt{\frac{k^{\prime}}{D_{\mathrm{e}}}}=F C_{\mathrm{e}}^{\delta} \sqrt{k_{\mathrm{s}} C_{\mathrm{s}} \bar{D}_{\mathrm{e}}}
$$

On the contrary, when the diffusion rate is predominant in comparison with the scavenging rate, the photocurrent is expressed as follows;

$$
i_{\mathrm{p}}{ }^{\prime}=F C_{\mathrm{e}}{ }^{\delta} k_{\mathrm{s}} C_{\mathrm{s}} r
$$

In Eq. (7) the photocurrent should be linearly proportional to the square root of the scavenger concentration. Fig. 1 shows the plot of the photocurrent $v s$, the square root of the DMSO concentration in the PC solution. The

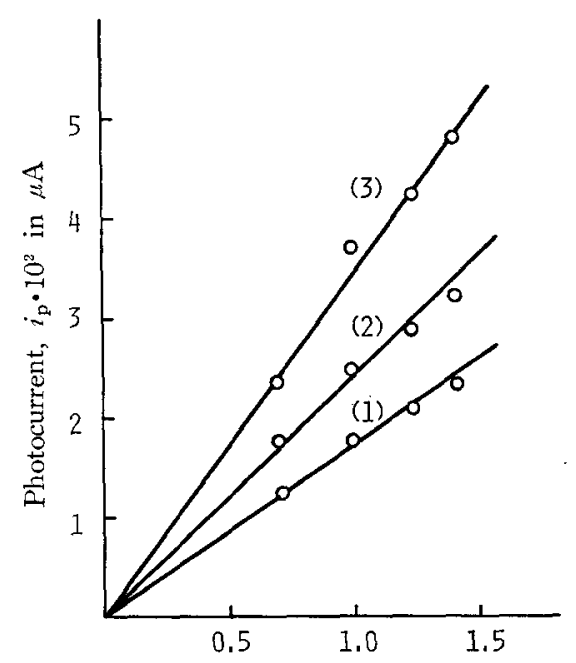

Square root of the concentration of DMSO, $C_{\mathrm{s}}{ }^{1 / 2}$ in $M^{1 / 2}$

Fig. 1 Effect of the scavenger concentration on the photocurrent at different potentials in PC solution containing $0.1 \mathrm{M}$ $\mathrm{NaClO}_{4}$. Scavenger DMSO.
(1) $-1.4 \mathrm{~V}$ vs. S.C.E., (2) $-1.5 \mathrm{~V}$ and $(3)-1.6 \mathrm{~V}$ 
photocurrent was measured at the applied potential of $-1.4,-1.5$ and $-1.6 \mathrm{~V}$ vs. SCE. In each plot the linearity is satisfactory in good agreement with the theory (Eq. (7)).

The reaction product of DMSO with the solvated electron in this process is assumed to be dimethylsulfinylcarbanion as has been confirmed by the radiation chemistry studies ${ }^{14}$. The reaction is given as follows;

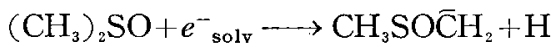

The following coupled chemical reactions of Reaction (9) have been discussed in detail by the present authors ${ }^{15}$.

\subsection{Field effect on the photocurrent}

The field effect on the photocurrent has been discussed by Delahay and Srinivasan ${ }^{7)}$ and Delahay et al..$^{8)}$ on the basis of Fowler's theory, and they proved the quadratic dependence of the photocurrent on the field strength in the scavenging reaction of $e^{-}$aq with the hydrogen ion. The relation is simply expressed as follows;

$$
i_{\mathrm{p}}=A \phi^{2}
$$

where $\phi$ denotes the electrode potential and $A$ the constant.

Later, Brodsky and Gurevich" have developed the quantum mechanical theory of the photoelectron emission from the microscopically homogeneous metal surface into the solution, and they derived the law of five halves, $i$. e.,

$$
i_{\mathrm{p}}=A^{\prime}\left(h \nu-h \nu_{0}(0)-e \phi\right)^{5 / 2}
$$

where $A^{\prime}$ is the constant characterizing the electrode metal considered, $h \nu$ the photon energy, $\nu_{0}(0)$ the threshold frequency of the photoelectron emission at $\phi=0$.

Fig. 2 shows the plot of $i_{\mathrm{p}}{ }^{0.5}$ or $i_{\mathrm{p}}{ }^{0.4}$ vs. the electrode potential in the case of DMSO solution containing $0.1 \mathrm{M}$ sodium perchlorate. It is noteworthy that in this case PC was not used as the solvent, and DMSO used as the medium plays a role of the scavenger at the same time. Both two plots give fairly good straight lines and it seems that there is no noticeable difference between their linearities.

It has been pointed out ${ }^{9>11}$ that the potential in the diffuse double layer affects the photoelectron emission in a rather diluted electrolyte solution as shown in the following equation;

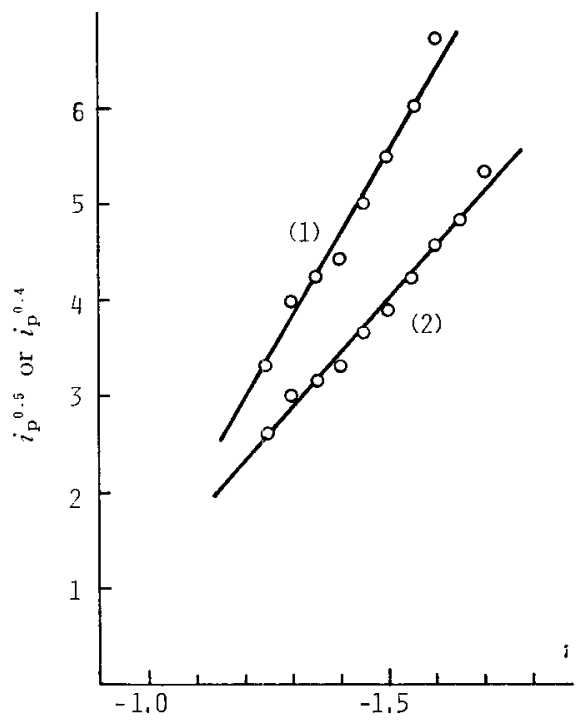

Electrode potential, $\phi \mathrm{V}$ vs. NCE

Fig. 2 Dependence of the electrode potential on the photocurrent in DMSO solutions containing $0.1 M \mathrm{NaClO}_{4}$. (1) Plot of $i_{\mathrm{p}}{ }^{0.5} v s . \phi$ and (2) $i_{\mathrm{p}}{ }^{0.4} v s . \phi$

$$
i_{\mathrm{p}}=A^{\prime}\left\{h \nu-h \nu_{0}(0)-e\left(\phi-\psi_{2}\right)\right\}^{5 / 2}
$$

where $\psi_{2}$ denotes the potential difference in the diffuse double layer.

The value of $\psi_{2}$-potential was calculated by the Gouy-Chapman equation from the differential capacity data measured by the impedance bridge. The results obtained in the DMSO solution by changing the sodium perchlorate concentration are illustrated in Fig. 3, and these data are used for the double layer correction shown in Fig. 4.

Figs. 4 and 5 show the plot of $i_{\mathrm{p}}{ }^{0.4}$ vs. the electrode potential with and without the double layer correction, respectively, when the concentration of sodium perchlorate was reduced from 0.1 to 0.01 and $0.001 M$ in the DMSO solution. It is noteworthy that the extrapolated straight lines in Fig. 4 intersect each other at a point on the zero current abscissa, and give the value of the threshold potential of $-0.66 \mathrm{~V}$ vs. NCE.

Considering the following energy $\mathrm{cycle}^{16)}$, where an electron emitted from the metal to vacuum passes to solution through the vacuumsolution interface and finally returns to the metal through the metal-solution interface, 


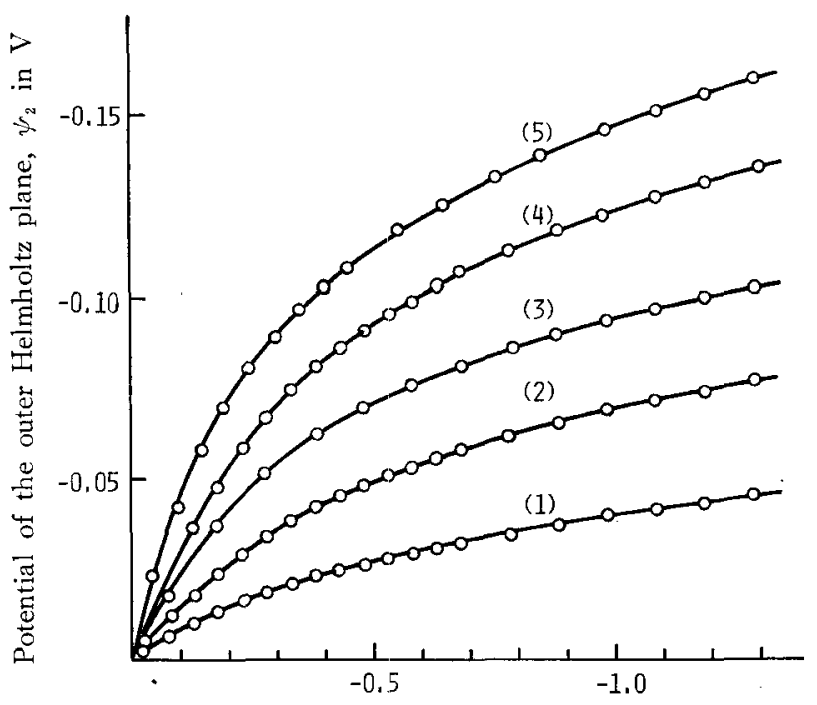

Rational potential, $\left(\phi-\phi_{\mathrm{p} z \mathrm{c}}\right) \mathrm{V}$ 's. NCE

Fig. 3 Potential of the outer Helmholtz plane as a function of the rational potential in DMSO solution.

(1) $1.66 M$ in $\mathrm{NaClO}_{4}$, (2) $0.3 M$, (3) $0.1 M$,

(4) $0.01 M$ and (5) $0.001 M$

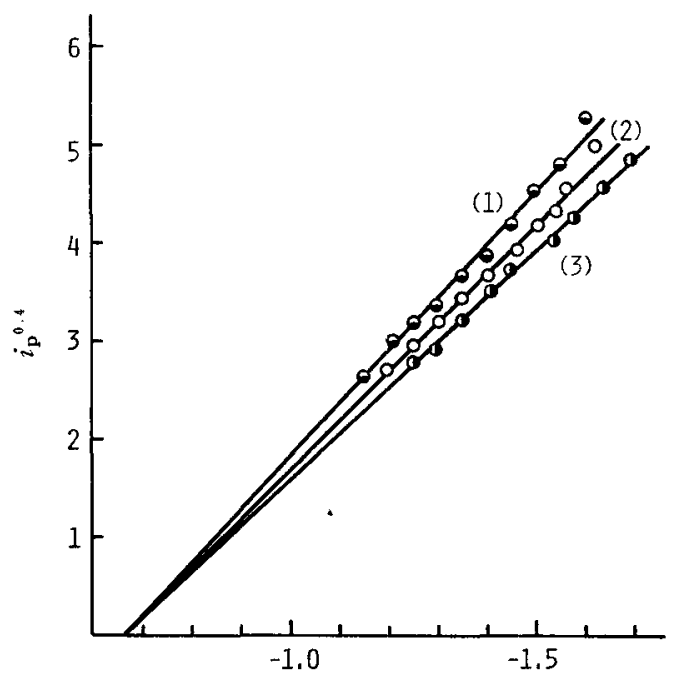

Electrode potential corrected for $\psi_{2},\left(\phi-\psi_{2}\right)$ in $\mathrm{V}$ vs. $\mathrm{NCE}$

Fig. 4 Effect of the double layer correction on the relationship between the photocurrent and the electrode potential in DMSO solution.

(1) $0.1 M$ in $\mathrm{NaClO}_{4}$, (2) $0.01 \mathrm{M}$ and (3) $0.001 \mathrm{M}$

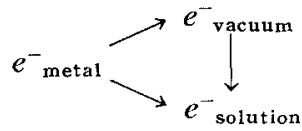

we obtain Eq. (14) by assuming that there is no spacific adsorption at the metal-solution interface, $i$. $e$.,

$$
W_{\mathrm{m}-\mathrm{s}}=W_{\mathrm{m}-\mathrm{v}}+V_{\mathrm{m}-\mathrm{s}}-U_{\mathrm{e}-\mathrm{s}}
$$

where $W_{\mathrm{m}-\mathrm{s}}$ is the work function of the electron emission from the metal into the solution, $W_{\mathrm{m}-\mathrm{v}}$ the work function from the metal into vacuum, $V_{\mathrm{m}-\mathrm{s}}$ the Volta potential difference between the metal and solution, and $U_{\mathrm{e}-\mathrm{s}}$ the real free energy of the solvation of the electron.

The energy quantum, $h \nu_{0}(0)$, in Eq. (11) or (12) can be substituted by the work function, $W_{\mathrm{m}-\mathrm{s}}$ at $\phi=0, i$. e.,

$$
i_{\mathrm{p}}=A^{\prime}\left\{h \nu-\left(W_{\mathrm{m}-\mathrm{s}}\right)_{\phi=0}-e \phi\right\}^{5 / 2}
$$

At the threshold potential, $\phi_{0}$, where the photocurrent becomes null, Eq. (15) can be rewritten as;

$$
h \nu-\left(W_{\mathrm{m}-\mathrm{s}}\right)_{\phi=0}-e \phi_{0}=0
$$

In Eqs. (15) and (16) the expression of the potential referred to the point of

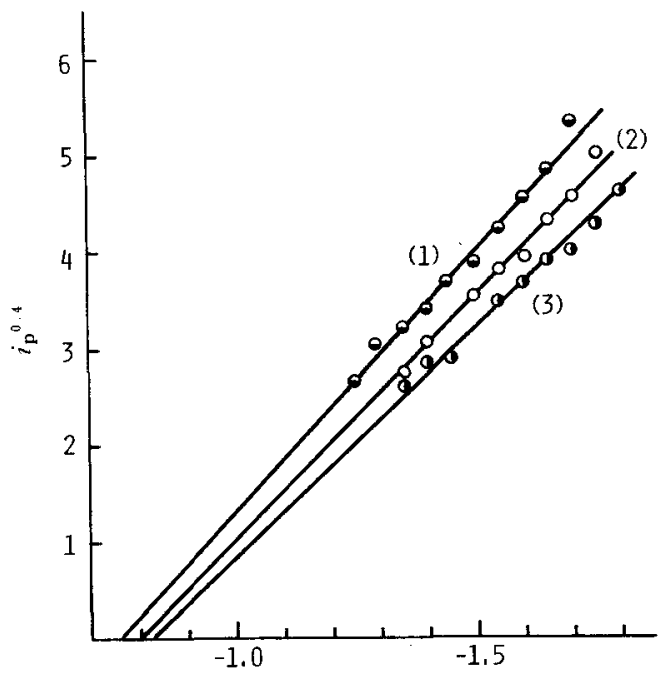

Electrode potential, $\phi \mathrm{V}$ vs. NCE

Fig. 5 Relationship between the photocurrent and the electrode potential. (1) Plot of $i_{\mathrm{p}}{ }^{0.4}$ vs. $\phi$ in DMSO solution containing $0.1 M \mathrm{NaClO}_{4}$, (2) $0.01 M$, and (3) $0.001 \mathrm{M}$ 
zero charge (pzc)seems to be preferable from the rigorous definition of the threshold frequency.

Thus, from Eqs. (14) and (16) we obtain the following expression;

$$
U_{\mathrm{e}-\mathrm{s}}^{r}-\left(V_{\mathrm{m}-\mathrm{s}}\right)_{\phi_{\mathrm{p} z \mathrm{c}}}=W_{\mathrm{m}-\mathrm{v}}-h \nu+e\left(\phi_{0}-\phi_{\mathrm{pzc}}\right)
$$

In applying Eq. (17) to the present case it is noteworthy that DMSO used as the solvent plays a role of the scavenger of the electron at the same time. The numerical values estimated for the terms in the r.h.s. of Eq. (17) are as follows;

$$
\begin{aligned}
W_{\mathrm{Hg} \text {-vacuum }}= & 4.52 \mathrm{eV} \\
h \nu & 3.50 \mathrm{eV} \\
e \phi_{0} & =-0.69 \mathrm{eV} \text { (the potential value } \\
& \text { is referred to S.C.E.) } \\
\phi_{\mathbf{p z c}} & =-0.35 \mathrm{~V} \text { os. S. C. E. }
\end{aligned}
$$

where the value of $h \nu$ corresponds to the wavelength of $\sim 350 \mathrm{~nm}$, and this value seems to be appropriate in view of the experimental condition which uses the light source from a xenon lamp, the filter to pass $250 \sim 380 \mathrm{~nm}$ light and the pyrex glass cell with a water jacket.

Using these data we obtain Eq. (18) as follows;

$$
U_{\mathrm{e}-\mathrm{s}}-\left(V_{\mathrm{m}-\mathrm{s}}\right)_{\phi_{\mathrm{pze}}}=0.74 \mathrm{eV}
$$

Eq. (18) shows that the value of $U_{\mathrm{e}-\mathrm{s}}$ can be estimated when the value of $\left(V_{\mathrm{m}-\mathrm{s}}\right)_{\phi_{\mathrm{p} r \mathrm{c}}}$ is known, or vice versa.

The value of $0.74 \mathrm{eV}$ is fairly small compared with the value of $1.57 \mathrm{eV}$ obtained for the photo-electron emission into the aqueous solution $^{11}$. No decisive conclusion can be given at this stage because the value of the Volta p.d. between mercury and DMSO is not known, and the liquid junction potential is not corrected for the estimation of the potential value, but the smaller value in the DMSO solution compared with the value in an aqueous solution seems to be self-consistent when one compares the values of the dipole moments of water and DMSO molecules.

In connection with this problem Rotenberg $e t$ $a l .{ }^{11}$ have found that the value of $U_{\mathrm{e}-\mathrm{s}}$ in water is $1.57 \mathrm{eV}$. In their evaluation the value of $V_{\mathrm{m}-\mathrm{s}}$ in $\mathrm{Eq}$. (18) has been neglected. The value can be estimated from the data obtained by Randles ${ }^{17)}$ and Grahame et al. ${ }^{187}$ to be $-0.26 \mathrm{~V}$. When this value is taken into account, the above mentioned value of $1.57 \mathrm{eV}$ should be corrected to be $1.31 \mathrm{eV}$. This value is small compared with the hydration energy of the electron as great as $1.75 \mathrm{eV}$ which has been determined by the radiolysis study ${ }^{13)}$. Rotenberg et $a l$. have tried to elucidate the discrepancy between $1.57 \mathrm{eV}$ and $1.75 \mathrm{eV}$ by assuming that the emitted electrons are not necessarily hydrated prior to the scavenging reaction, in other words, the fast electrons can also participate in the scavenging reaction. If this assumption is valid, there arises a contradiction with our theory described in the first half of this paper, which involves the solvation of the thermalized electron, the slow electron, its semi-infinite diffusion and the followed scavenging reaction. Moreover, the similar mechanism has already been proved in the aqueous medium by Barker ${ }^{3)}$ and also by the present authors ${ }^{6}$. The latter paper has treated the effects of the ionic strength and dielectric constant of the medium on the rate of the scavenging reaction of the hydrated electrons in the homogeneous solution phase from the basis of the $B r \phi n s t e d$ theory. The details of this problem will be discussed in the following paper.

\section{Conclusion}

A general theory on the photocurrent has been developed, where it is assumed that emitted electrons from the irradiated electrode are thermalized, solvated and then react with scavenger at a certain distance apart from the electrode surface in solutions.

The quadratic dependence of the photocurrent on the concentration of scavenger and the law of five halves on the field effect were proved experimentally in the cases of the DMSO solution and PC solution containing DMSO, in both cases DMSO playing a role of the scavenger.

This result suggests that around the irradiated dropping mercury electrode DMSO molecules are reduced by the solvated electrons and then methylsulfinylcarbanions are formed.

In relation to the physical significance of the threshold potential the real free energy of the solvation of the electron, $U_{\mathbf{e}^{-s}}$, was evaluated by using the data of the energy quantum of the incident light, the work function of the electron from the metal into vacuo, and the Volta p. d. 
between the metal and the solution, $V_{\mathrm{m}-\mathrm{s}}$.

The value of $\left\{U_{\mathrm{e}-\mathrm{s}}-\left(V_{\mathrm{m}-\mathrm{s}}\right)_{\phi_{\mathrm{pzc}}}\right\}$ estimated in DMSO was about a half of that in water.

The $U_{\mathrm{e}-\mathrm{s}}$ evaluated by the photoelectrochemical emission method was smaller than that determined by the radiolysis technique ( 1.75 $\mathrm{eV}$ ). The discussion of this discrepancy is remained for the future.

$$
<\text { E 412> (Received Feb. 27, 1975) }
$$

\section{References :}

1) M. Heyrovsky, Proc. Roy. Soc. London, Ser. A. 301, 411 (1967).

2) H. Berg, H. Schweiss, E. Stutter, K. Weller, J. Electroanal. Chem. 15, 415 (1967).

3) G.C. Barker, A.W. Gardner, D.S. Sammon, $J$. Electrochem. Soc. 113, 11.82 (1966).

4) M.S. Matheson, J. Rabani, J. Phys. Chem. 89, 1324 (1965).

5) L.M. Dorfman, I.A. Taub, J. Amer. Chem. Soc. 85, 2370 (1963).

6) K. Yamashita, H. Imai, Bull. Chem. Soc. Japan 45, 628 (1972).
7) P. Delahay, V.S. Srinivasan, J. Phys. Chem. 70, 420 (1966)

8) V.P. Sharma, P. Delahay, G.G. Susbielles, G. Tessari, J. Electroanal. Chem. 16, 285 (1968).

9) A.M. Brodsky, Yu. Ya. Gurevich, Electrochim. Acta 13, 1245 (1968); See also, A.M. Brodsky, Yu. Ya. Gurevich, S.V. Sheberstov, J. Electroanal. Chem. 32, 353 (1971).

10) Yu. V. Pleskov, Z.A. Rotenberg, J. Electroanal. Chem. 20, 1 (1969).

11) Z.A. Rotenberg, V.I. Lakomor, Yu. V. Pleskov, J. Electroanal, Chem. 27, 403 (1970).

12) G. Bomchil, D.J. Schiffrin, J.T. D'alessio, J. Electroanal. Chem. 25, 107 (1970).

13) J. Baxendale, Rad. Research Suppl. 4, 139 (1964).

14) T.K. Cooper, D.C. Walker, Can. J. Chem. 49, 2248 (1971).

15) H. Imai, K. Yamashita, Chem. Letters (CSJ) 423 (1972).

16) A.N. Frumkin, J.Electroanal. Chem. 9, 173 (1965).

17) J. Randles, Trans. Faraday Soc. 52, 1573 (1956).

18) D.C. Grahame, E. Coffin, J. Cummings, M.A. Poth, J. Amer. Chem. Soc. 74, 1207 (1952). 